\title{
Finite Groups Which Are the Union of Autocentralizers of Some Automorphisms
}

\author{
Esmat Alamshahi, ${ }^{1}$ Mohammad Reza R. Moghaddam $\mathbb{D},{ }^{1,2,3}$ \\ and Mohammad Amin Rostamyari ${ }^{2}{ }^{2}$ \\ ${ }^{1}$ Department of Mathematics, Mashhad Branch, Islamic Azad University, Mashhad, Iran \\ ${ }^{2}$ Department of Mathematics, Khayyam University, Mashhad, Iran \\ ${ }^{3}$ Department of Pure Mathematics, Centre of Excellence in Analysis on Algebraic Structures (CEAAS), \\ Ferdowsi University of Mashhad, P.O.Box 1159, Mashhad, 91775, Iran \\ Correspondence should be addressed to Mohammad Reza R. Moghaddam; rezam@ferdowsi.um.ac.ir
}

Received 1 September 2021; Accepted 11 October 2021; Published 18 January 2022

Academic Editor: Naihuan Jing

Copyright (c) 2022 Esmat Alamshahi et al. This is an open access article distributed under the Creative Commons Attribution License, which permits unrestricted use, distribution, and reproduction in any medium, provided the original work is properly cited.

\begin{abstract}
Let $\alpha$ be an automorphism of a given group $G$; then, $C_{G}(\alpha)=\{g \in G \mid \alpha(g)=g\}$ is called the autocentralizer of $\alpha$ in $G$. In this work, we study finite groups $G$, which can be written as the union of autocentralizers of some automorphisms of $G$. In particular, if a group $G$ is 5 - $A$-centralizers, then we determine the absolute central quotient $G / L(G)$ of $G$, where $A=$ Aut $(G)$. Finally, it is shown that autocommutative property of a group is equivalent to the one, in which every $A$-centralizer of its nontrivial elements is abelian.
\end{abstract}

\section{Introduction}

Let $A=\operatorname{Aut}(G)$ be the group of all automorphisms of a given group $G$ and $\alpha \in$ A. The autocentralizer or A-centralizer of $\alpha$ in $G$ is the subgroup of $G$ consisting of all elements of $G$ which are fixed by $\alpha$ and denoted by $C_{G}(\alpha)$, i.e.,

$$
C_{G}(\alpha)=\left\{g \in G \mid g^{\alpha}=\alpha(g)=g\right\} .
$$

Also, we denote the set of all such autocentralizes in $G$ by $C_{G}(A)$. Note that, if $C_{G}(\alpha)=\{e\}$, where $e$ is the identity element of $G$, then $\alpha$ is called fixed-point-free automorphism. We remark that, for the identity automorphism id. of $A$, we have $G=C_{G}(\mathrm{id})$, and hence, $G \in C_{G}(A)$.

For any element $g \in G$ and $\alpha \in \mathrm{A}$, the element,

$$
[g, \alpha]=g^{-1} g^{\alpha}=g^{-1} \alpha(g),
$$

is the autocommutator of $g$ and $\alpha$. Clearly, if $\alpha=\phi_{x}$ is the inner automorphism induced by the element $x$ of $G$, then the autocommutator $\left[g, \phi_{x}\right]$ coincides with the ordinary commutator $[g, x]$. The set $L(G)$, consisting of all elements $g$ in
$G$ which are fixed by every automorphism of $G$, is a central characteristic subgroup of $G$, which is called absolute centre or the autocentre of $G$ (see [1-5], for more information). Hence, we may write

$$
L(G)=\{g \in G:[g, \alpha]=1, \forall \alpha \in \mathrm{A}\}=\underset{\alpha \in \mathrm{A}}{\cap} C_{G}(\alpha) .
$$

We denote the number of distinct autocentralizers of automorphisms in $G$ by $\left|C_{G}(A)\right|$, and now a question arises that "how many distinct autocentralizers can a group have?"

This article is motivated by providing some answers to the above question.

\section{Preliminary Results}

It is clear that, in a given group $G$, there may exist an element $x$, which is not fixed by none of nontrivial automorphisms of $G$. For example, consider cyclic groups of order $p$, for odd prime $p$.

In the present article, we consider all finite groups which do not have the above property, so the following result is obvious. 
Lemma 1. A group $G$ is the union of autocentralizers of all nontrivial automorphisms of $A$ in $G, L(G)$, i.e., $G=\cup_{i d \neq \alpha \in A} C_{G, L(G)}(\alpha)$.

Proof. Clearly, $\quad \cup_{\mathrm{id} \neq \alpha \in \mathrm{A}} C_{G}, L(G)(\alpha) \subseteq G$. Now, if $x \in G, L(G)$, then, by the above assumption, there exists $\alpha \in \mathrm{A}$, such that $x \in C_{G}, L(G)(\alpha)$. Therefore, $G \subseteq \cup_{\mathrm{id} \neq \alpha \in \mathrm{A}} C_{G}, L(G)(\alpha)$, which completes the proof.

Remark 1. Note that if $\left|C_{G}(A)\right|=1$, then (3) implies that $L(G)=G$, so $[x, \alpha]=1$, for all $x \in G$ and $\alpha \in$ A. Hence, $A=\{\mathrm{id}\}$, and consequently, $G=\langle 1\rangle$ or $\mathbb{Z}_{2}$. Clearly, the converse is also true. Therefore, $\left|C_{G}(A)\right|=1$ if and only if $G=\langle 1\rangle$ or $\mathbb{Z}_{2}$.

Lemma 2. Let $G$ be a nontrivial group except $\mathbb{Z}_{2}$; then, $\left|C_{G}(A)\right| \geq 4$.

Proof. By Remark 1, $\left|C_{G}(A)\right| \neq 1$. If $\left|C_{G}(A)\right|=2$, then $G$ is the proper subgroup of itself, which is impossible. Suppose that $\left|C_{G}(A)\right|=3$; then, $C_{G}(A)=\left\{G, C_{G}\left(\alpha_{1}\right), C_{G}\left(\alpha_{2}\right)\right\}$, for some automorphisms $\alpha_{1}$ and $\alpha_{2} \in \mathrm{A}$, where $C_{G}\left(\alpha_{1}\right)$ and $C_{G}\left(\alpha_{2}\right)$ are proper subgroups of $G$. Therefore, $G=C_{G}\left(\alpha_{1}\right) \cup C_{G}\left(\alpha_{2}\right)$, which is impossible, since a group cannot be written as the union of two proper subgroups. Thus, $\left|C_{G}(A)\right| \geq 4$.

A group $G$ is called $n$-A-centralizer if $\left|C_{G}(A)\right|=n$ (see also [6]).

\section{Example 1}

(i) Consider $\quad D_{8}=\left\langle x, y \mid x^{4}=y^{2}=1, x^{y}=x^{-1}\right\rangle$, the Dihedral group of order 8; then, clearly, the set of all automorphisms of $D_{8}$ is

$$
\alpha_{i j}: \begin{cases}x \mapsto x^{i}, & i=1,3, \\ y \mapsto x^{j} y, & j=1,2,3,4 .\end{cases}
$$

Thus, one can calculate that

$$
\begin{aligned}
& C_{D_{8}}\left(\alpha_{11}\right)=C_{D_{8}}\left(\alpha_{12}\right)=C_{D_{8}}\left(\alpha_{13}\right)=\langle x\rangle, \\
& C_{D_{8}}\left(\alpha_{31}\right)=C_{D_{8}}\left(\alpha_{33}\right)=\left\{1, x^{2}\right\}, \\
& C_{D_{8}}\left(\alpha_{14}\right)=D_{8}, \\
& C_{D_{8}}\left(\alpha_{32}\right)=\left\{1, x^{2}, x y, x^{3} y\right\}, \\
& C_{D_{8}}\left(\alpha_{34}\right)=\left\{1, x^{2}, y, x^{2} y\right\} .
\end{aligned}
$$

Therefore, $D_{8}$ is 4 -A-centralizer, as $C_{D_{8}}\left(\alpha_{31}\right)$ and $C_{D_{8}}\left(\alpha_{32}\right)$ are not distinct.

Similarly, one can easily see that $\left|C_{Q_{8}}(A)\right|=4$.

(ii) Consider $S_{3}=\left\langle x, y \mid x^{3}=y^{2}=1, x^{y}=x^{-1}\right\rangle$, the symmetric group of order 6 ; then, one can calculate that $S_{3}$ has $5 \mathrm{~A}$-centralizers as follows:

$$
S_{3},\{1, y\},\left\{1, x, x^{2}\right\},\left\{1, x^{2} y\right\},\{1, x y\}
$$

\section{Counting A-Centalizers in Groups}

For an arbitrary element $x$ of a given group $G$, the set $C_{G}(x)=\left\{y \in G:[y, x]=\left[y, \phi_{x}\right]=1\right\}$, where $\phi_{x}$ is the inner automorphism of $G$, is called the centralizer of $x$ in $G$. The set of all such centralizers in $G$ is denoted by Cent $(G)$. Clearly, $G$ is abelian if and only if $|\operatorname{Cent}(G)|=1$.

Many authors have studied the influence of the number of centralizers of a finite group $G$ on the structure of the group. In 1994, Belcastro and Sherman [7] proved that $\mid$ Cent $(G) \mid \geq 4$, for any nonabelian finite group $G$. They also showed that $G$ has 4 centralizers if and only if $G / Z(G) \cong$ $C_{2} \times C_{2}$ and $G$ has 5 centralizers if and only if $G / Z(G) \cong$ $C_{3} \times C_{3}$ or $S_{3}$. In [8], Ashrafi proved that if $G$ has 6 centralizers, then $G / Z(G) \cong D_{8}, A_{4}, C_{2} \times C_{2} \times C_{2} \quad$ or $C_{2} \times C_{2} \times C_{2} \times C_{2}$, where $A_{4}$ is the alternating group of degree 4 .

In this section, we compute $\left|C_{G}(A)\right|$ under certain conditions on the group $G$.

Proposition 1. Let $D_{2 n}$ be the Dihedral group of order 2n; then,

$$
\left|C_{D_{2 n}}(A)\right| \mid= \begin{cases}n+2, & n \text { is odd } \\ \frac{n}{2}+2, & n \text { is even. }\end{cases}
$$

Proof. Clearly, for $n \geq 3$, the group $D_{2 n}$ has the following presentation:

$$
\begin{aligned}
D_{2 n} & =\left\langle x, y \mid x^{n}=y^{2}=1, x^{y}=x^{-1}\right\rangle \\
& =\left\{1, x, \ldots, x^{n-1}, y, x y, \ldots, x^{n-1} y\right\} .
\end{aligned}
$$

Then, $A=\left\{\alpha_{i j}:\left\{\begin{array}{l}x \mapsto x^{i} \\ y \mapsto x^{j} y\end{array} \mid \operatorname{gcd}(i, n)=1,0 \leq j \leq n-1\right\}\right.$, so $C_{D_{2 n}}\left(\alpha_{10}\right)=D_{2 n}$. Assume that $n$ is odd; then, we have the following $A$-centralizers:

(i) $C_{D_{2 n}}\left(\alpha_{1 j}\right)=\langle x\rangle, \quad$ as $\quad\left[x^{k}, \alpha_{1 j}\right]=x^{-k} x^{k}=1$, $\left[y, \alpha_{1 j}\right]=y^{-1} x^{j} y=x^{-j} \neq 1, \quad$ and $\left[x^{k} y, \alpha_{1 j}\right]=y^{-1} x^{-k} x^{k} x^{j} y=x^{-j} \neq 1$, for every $0<k \leq n-1$.

(ii) $C_{D_{2 n}}\left(\alpha_{i 0}\right)=\{1, y\}, \quad$ as $\quad\left[x^{k}, \alpha_{i 0}\right]=x^{-k} x^{i k} \neq 1$, $\left[y, \alpha_{i 0}\right]=y^{-1} y=1$, and $\left[x^{k} y, \alpha_{i 0}\right]=y^{-1} x^{-k} x^{i k} y=x^{k-i k} \neq 1, \quad$ for every $0<k \leq n-1$ and even $i$.

(iii) $C_{D_{2 n}}\left(\alpha_{21}\right)=\left\{1, x^{n-1} y\right\}$, as $\left[x^{k}, \alpha_{21}\right]=x^{-k} x^{2 k} \neq 1$, for every $0<k \leq n-1,\left[y, \alpha_{21}\right]=y^{-1} x y=x^{-1} \neq 1$, and $\left[x^{s} y, \alpha_{21}\right]=y^{-1} x^{-s} x^{2 s} x y=x^{s+1}=1$, when $s=n-1$.

By similar argument, one has $C_{D_{2 n}}\left(\alpha_{22}\right)=\{1$, $\left.x^{n-2} y\right\}, C_{D_{2 n}} \quad\left(\alpha_{23}\right)=\left\{1, x^{n-3} y\right\}, \ldots, C_{D_{2 n}}\left(\alpha_{2(n-1)}\right)$ $=\{1, x y\}$, so $\left|C_{D_{2 n}}(A)\right|=n+2$ if $n$ is odd.

Now, assume that $n$ is even; then, we have the following cases 
(iv) $C_{D_{2 n}}\left(\alpha_{1 j}\right)=\langle x\rangle, \quad$ as $\quad\left[x^{k}, \alpha_{1 j}\right]=x^{-k} x^{k}=1$, $\left[y, \alpha_{1 j}\right]=y^{-1} x^{j} y=x^{-j} \neq 1$, and $\left[x^{k} y, \alpha_{1 j}\right]=y^{-1} x^{-k} x^{k} x^{j} y=x^{-j} \neq 1, \quad$ for $\quad$ every $0<k \leq n-1$.

(v) $C_{D_{2 n}}\left(\alpha_{(n-1) 0}\right)=\left\{1, y, x^{(n / 2)}, x^{(n / 2)} y\right\}, \quad$ as $\left[x^{k^{n}}, \alpha_{(n-1) 0}\right]=x^{-k} x^{n k-k}=1, \quad$ when $\quad k=n / 2$, $\left[y, \alpha_{(n-1) 0}\right]=y^{-1} y=1, \quad$ and $\left[x^{k} y, \alpha_{(n-1) 0}\right]=y^{-1} x^{-k} x^{n k-k} y=x^{2 k-n k}=1$, when $k=(n / 2)$.

Using similar argument for $C_{D_{2 n}}\left(\alpha_{(n-1) j}\right)$, when $j$ is even, we have the following $A$-centralizers $C_{D_{2 n}}\left(\alpha_{(n-1) 2}\right)$ $=\left\{1, x y, x^{(n / 2)}, x^{(n / 2)+1} y\right\}, \quad C_{D_{2 n}}\left(\alpha_{(n-1) 4}\right)$ $=\left\{1, x^{2} y, x^{(n / 2)}, x^{(n / 2)+2} y\right\}, \quad \ldots, C_{D_{2 n}}\left(\alpha_{(n-1)(n-2)}\right)$ $=\left\{1, x^{(n / 2)-1} y, x^{(n / 2)}, x^{n-1} y\right\}$, so $\left|C_{D_{2 n}}(A)\right|=(n / 2)+2$ if $n$ is even.

The following result of [9] is useful in our further investigations.

Theorem 1 (see Theorem 3.1 in [9]). Let $G$ be a group with $G / L(G) \cong C_{p} \times C_{p}$, for any prime number $p$. Then, $G$ is isomorphic to one of the following groups:

(1) $C_{p} \times C_{p}$

(2) $C_{p} \times C_{p} \times C_{2}$ (p is odd)

(3) $C_{4} \times C_{2}$

(4) $D_{8}$

(5) $Q_{8}$

(6) $\left\langle x, y: x^{4}=y^{4}=1, x^{y}=x^{-1}\right\rangle$

Using the above theorem, we have the following.

Theorem 2. Let $G$ be a group such that $G / L(G) \cong C_{p} \times C_{p}$, for any prime number $p$. Then, $\left|C_{G}(A)\right|=p+2$.

Proof. Let $p=2$ and $G / L(G) \cong C_{2} \times C_{2}$; then, Proposition 1 implies

that

$G \cong C_{2} \times C_{2}=\left\langle x, y: x^{2}=1, y^{2}=1,[x, y]=1\right\rangle . \quad$ Clearly, $A=\operatorname{Aut}\left(C_{2} \times C_{2}\right) \cong S_{3}$, and it is easily checked that $\left|C_{C_{2} \times C_{2}}(A)\right|=4$.

Assume $G=C_{4} \times C_{2}=\left\langle x, y: x^{4}=y^{2}=1,[x, y]=1\right\rangle$; then, clearly,

$$
\operatorname{Aut}\left(C_{4} \times C_{2}\right)=\left\{\alpha_{i j}:\left\{\begin{array}{l}
x \mapsto x \text { or } x^{3} \text { or } x y \text { or } x^{3} y \\
y \mapsto y \text { or } x^{2} y
\end{array}\right\} .\right.
$$

Hence, one can calculate that the group $C_{4} \times C_{2}$ has the following $A$-centralizers:

$$
C_{4} \times C_{2},\langle x\rangle,\left\{1, x^{2}, x y, x^{3} y\right\},\left\{1, x^{2}, y, x^{2} y\right\} .
$$

In the cases $G \cong D_{8}$ and $Q_{8}$, example 1 and Proposition 1 show that $G$ is 4 - $A$-centralizer.

If $G=\left\langle x, y: x^{4}=y^{4}=1, x^{y}=x^{-1}\right\rangle$, then with relatively simple and long calculations, we obtain that $G$ has the following $A$-centralizers:

$$
\begin{array}{r}
G,\left\{1, x^{2}, y^{2}, x^{2} y^{2}, x, x^{3}, x y^{2}, x^{3} y^{2}\right\}, \\
\left\{1, x^{2}, y^{2}, x^{2} y^{2}, y, x^{2} y, y^{3}, x^{2} y^{3}\right\}, \\
\left\{1, x^{2}, y^{2}, x^{2} y^{2}, x y, x^{3} y, x y^{3}, x^{3} y^{3}\right\} .
\end{array}
$$

Now, let $G \cong C_{p} \times C_{p} \times C_{2}$ and $p$ be an odd prime number. Then, it is clear that $\left|C_{G}(\alpha)\right|=2,2 p$ or $2 p^{2}$, for every $\alpha \in A$. Thus, we have the following $A$-centralizers:

$$
\begin{aligned}
G= & \left\langle x, y, z: x^{p}=y^{p}=z^{2}=1,[x, y]=[x, z]=[y, z]=1\right\rangle, \\
& \cdot\{1, z\}, \\
& \cdot\left\{1, z, x, x^{2}, \ldots, x^{p-1}, z x, z x^{2}, \ldots, z x^{p-1}\right\}, \\
& \cdot\left\{1, z, y, y^{2}, \ldots, y^{p-1}, z y, z y^{2}, \ldots, z y^{p-1}\right\}, \\
& \cdot\left\{1, z, x y^{i},\left(x y^{i}\right)^{2}, \ldots,\left(x y^{i}\right)^{p-1}, z x y^{i}, \ldots, z\left(x y^{i}\right)^{p-1}\right\},
\end{aligned}
$$

where $\quad 1 \leq i \leq p-1$, so $G$ has $p+2$ distinct $A$-centralizers.

The following theorem of [10] is needed to prove our next result.

Theorem 3 (see Theorem 1 in [10]). A group $G$ is the nontrivial union of three subgroups if and only if it is homomorphic to the Klein four-group.

Proposition 2. Let $G$ be a group; then, $\left|C_{G}(A)\right|=4$ if and only if $G / L(G) \cong C_{2} \times C_{2}$.

Proof. Using Theorem 1, it is enough to show that $\left|C_{G}(A)\right|=4$, which implies $G / L(G) \cong C_{2} \times C_{2}$.

Suppose $\quad\left|C_{G}(A)\right|=4$; then, $C_{G}(A)=\left\{G, C_{G}\left(\alpha_{1}\right), C_{G}\left(\alpha_{2}\right), C_{G}\left(\alpha_{3}\right)\right\}, \quad$ for some $\alpha_{1}, \alpha_{2}, \alpha_{3} \in A$. Hence, by Lemma $1, G=\cup_{i=1}^{3} C_{G}\left(\alpha_{i}\right)$. Consider $C_{G}\left(\alpha_{1} \alpha_{2}\right)$, which will be one of the $A$-centralizers $G, C_{G}\left(\alpha_{1}\right), C_{G}\left(\alpha_{2}\right)$, or $C_{G}\left(\alpha_{3}\right)$. If $C_{G}\left(\alpha_{1} \alpha_{2}\right)=G$, then $\alpha_{2}(x)=\alpha^{-1}(x)$, for all $x \in G$, so $C_{G}\left(\alpha_{1}\right)=C_{G}\left(\alpha_{2}\right)$, which is a contradiction.

Assume $C_{G}\left(\alpha_{1} \alpha_{2}\right)=C_{G}\left(\alpha_{1}\right)$, then for any $x \in C_{G}\left(\alpha_{1} \alpha_{2}\right)=C_{G}\left(\alpha_{1}\right)$, we have $\alpha_{1} \alpha_{2}(x)=\alpha_{1}(x)=x$. Therefore $\quad \alpha_{2}(x)=\alpha_{1}^{-1}(x)=x, \quad$ and hence, $C_{G}\left(\alpha_{1} \alpha_{2}\right)=C_{G}\left(\alpha_{1}\right) \subseteq C_{G}\left(\alpha_{2}\right)$, which is again a contradiction. Similarly, if $C_{G}\left(\alpha_{1} \alpha_{2}\right)=C_{G}\left(\alpha_{2}\right)$, then we have a contradiction, so $C_{G}\left(\alpha_{1} \alpha_{2}\right)=C_{G}\left(\alpha_{2} \alpha_{1}\right)$ must be equal with $C_{G}\left(\alpha_{3}\right)$.

Now, Theorem 3 implies that $\left(G / \cap_{i=1}^{3} C_{G}\left(\alpha_{i}\right)\right)=(G / L(G))$ is isomorphic with Klein fourgroup.

Theorem 4. Let $G$ be a finite group with $\left|C_{G}(A)\right|=5$; then, $G / L(G) \cong S_{3}, C_{3} \times C_{3}, D_{12}, C_{2} \times C_{6}, C_{3} \rtimes C_{4}$ or $A_{4}$.

Proof. Assume $\left|C_{G}(A)\right|=5$ and $C_{G}\left(\alpha_{i}\right)$ is $A$-centralizer, for some automorphisms $\alpha_{1}, \ldots, \alpha_{4}$ of $G$. Hence, Lemma 1 implies that $G=C_{G}\left(\alpha_{1}\right) \cup C_{G}\left(\alpha_{2}\right) \cup C_{G}\left(\alpha_{3}\right) \cup C_{G}\left(\alpha_{4}\right)$. 
Now, consider $C_{G}\left(\alpha_{1} \alpha_{2}\right)$, which should be one of $G, C_{G}\left(\alpha_{1}\right), C_{G}\left(\alpha_{2}\right), C_{G}\left(\alpha_{3}\right)$, or $C_{G}\left(\alpha_{4}\right)$. Thus, we have the following cases:

(a) If $C_{G}\left(\alpha_{1} \alpha_{2}\right)=G$, then $\alpha_{2}(x)=\alpha^{-1}(x)$, for all $x \in G$, so $C_{G}\left(\alpha_{1}\right)=C_{G}\left(\alpha_{2}\right)$, which is a contradiction.

(b) If $C_{G}\left(\alpha_{1} \alpha_{2}\right)=C_{G}\left(\alpha_{1}\right)$, then, for all $x \in C_{G}\left(\alpha_{1} \alpha_{2}\right)=C_{G}\left(\alpha_{1}\right), \quad$ we have $\alpha_{1} \alpha_{2}(x)=\alpha_{1}(x)=x$. Therefore, $\alpha_{2}(x)=\alpha_{1}^{-1}(x)=x, \quad$ and hence, $C_{G}\left(\alpha_{1} \alpha_{2}\right)=C_{G}\left(\alpha_{1}\right) \subseteq C_{G}\left(\alpha_{2}\right)$ and gives a contradiction.

Similarly, if $C_{G}\left(\alpha_{1} \alpha_{2}\right)=C_{G}\left(\alpha_{2}\right)$, then we obtain a contradiction, so $C_{G}\left(\alpha_{1} \alpha_{2}\right)$ can be equal to either $C_{G}\left(\alpha_{3}\right)$ or $C_{G}\left(\alpha_{4}\right)$.

(i) Assume that $C_{G}\left(\alpha_{1}\right), C_{G}\left(\alpha_{2}\right), C_{G}\left(\alpha_{1} \alpha_{2}\right)=C_{G}\left(\alpha_{1} \alpha_{2}\right)$, and $C_{G}\left(\alpha_{4}\right)$ are the $A$-centralizers of $G$. Then, using similar argument as in parts (a) and (b), we have $C_{G}\left(\alpha_{1} \alpha_{4}\right)=$ $C_{G}\left(\alpha_{2}\right)$ and $C_{G}\left(\alpha_{2} \alpha_{4}\right)=C_{G}\left(\alpha_{1}\right)$. On the contrary, $L(G) \subseteq C_{G}\left(\alpha_{1}\right) \cap C_{G}\left(\alpha_{2}\right)$. Thus, for all $x \in C_{G}\left(\alpha_{1}\right) \cap C_{G}\left(\alpha_{2}\right)$, we have

$$
\begin{aligned}
& \alpha_{1}(x)=\alpha_{2} \alpha_{4}(x)=x \Rightarrow \alpha_{2}^{-1}(x)=\alpha_{4}(x), \\
& \alpha_{2}(x)=\alpha_{1} \alpha_{4}(x)=x \Rightarrow \alpha_{2}^{-1}(x)=x,
\end{aligned}
$$

and hence, $x \in C_{G}\left(\alpha_{4}\right)$. Also, $\alpha_{1} \alpha_{2}(x)=\alpha_{1}(x)=x$ and $\quad \alpha_{2} \alpha_{1}(x)=\alpha_{2}(x)=x$ imply that $x \in C_{G}\left(\alpha_{1} \alpha_{2}\right)=C_{G}\left(\alpha_{2} \alpha_{1}\right)$. Therefore, $x \in L(G)$ and $L(G)=C_{G}\left(\alpha_{1}\right) \cap C_{G}\left(\alpha_{2}\right)$.

(ii) Assume that $C_{G}\left(\alpha_{1}\right), C_{G}\left(\alpha_{2}\right), C_{G}\left(\alpha_{1} \alpha_{2}\right)$, and $C_{G}\left(\alpha_{2} \alpha_{1}\right)$ are the $A$-centralizers of $G$. Then, similar argument as in part (i) implies that $L(G)=C_{G}\left(\alpha_{1}\right) \cap C_{G}\left(\alpha_{2}\right)$.

Hence, $\quad \cap_{i=1}^{4} C_{G}\left(\alpha_{i}\right)=L(G)=C_{G}\left(\alpha_{i}\right) \cap C_{G}\left(\alpha_{j}\right)$ $=C_{G}\left(\alpha_{i}\right) \cap C_{G}\left(\alpha_{j}\right) \cap C_{G}\left(\alpha_{k}\right)$, for $1 \leq i, j, k \leq 4$, when $\left|C_{G}(A)\right|=5$.

Now, for computing the value of $|L(G)|$, we show that if $C_{G}\left(\alpha_{i}\right)$ and $C_{G}\left(\alpha_{j}\right)$ are arbitrary distinct proper $A$-centralizers of $G$, for $1 \leq i \neq j \leq 4$; then,

$$
\frac{\left|C_{G}\left(\alpha_{i}\right)\right|\left|C_{G}\left(\alpha_{j}\right)\right|}{|G|} \leq|L(G)| \leq \frac{|G|}{6} \cdot(*) .
$$

Clearly,

$\left(\left|C_{G}\left(\alpha_{i}\right)\right|\left|C_{G}\left(\alpha_{j}\right)\right| /\left|C_{G}\left(\alpha_{i}\right) C_{G}\left(\alpha_{j}\right)\right|\right)=\left|C_{G}\left(\alpha_{i}\right) \cap C_{G}\left(\alpha_{j}\right)\right|$. As $C_{G}\left(\alpha_{i}\right) C_{G}\left(\alpha_{j}\right) \subseteq G$, we have $\left(1 /\left|C_{G}\left(\alpha_{i}\right) C_{G}\left(\alpha_{j}\right)\right|\right) \geq(1 /|G|)$. Therefore, $\left|C_{G}\left(\alpha_{i}\right) \cap C_{G}\left(\alpha_{j}\right)\right| \geq\left(\left|C_{G}\left(\alpha_{i}\right)\right|\left|C_{G}\left(\alpha_{j}\right)\right| /|G|\right)$ implies that $|L(G)| \geq\left(\left|C_{G}\left(\alpha_{i}\right)\right|\left|C_{G}\left(\alpha_{j}\right)\right| /|G|\right)$. On the contrary, one observes that

$$
\begin{aligned}
|G| & =\left|C_{G}\left(\alpha_{1}\right)\right|+\left|C_{G}\left(\alpha_{2}\right)\right|+\left|C_{G}\left(\alpha_{3}\right)\right|+\left|C_{G}\left(\alpha_{4}\right)\right|-3|L(G)| \\
& \geq 2|L(G)|+2|L(G)|+2|L(G)|+2|L(G)|-3|L(G)|=5|L(G)|,
\end{aligned}
$$

and hence, $(|G| /|L(G)|) \geq 5$. Assume $G / \mid L(G)=5$; then, $(G /|L(G)|)$ is cyclic and Theorem 2.2 of [9] implies that $G \cong$ $\mathbb{Z}_{5}$ or $\mathbb{Z}_{10}$, which both give contradictions as $\mathbb{Z}_{5}$ has fixed- point-free automorphism, and $\mathbb{Z}_{10}$ does not conform to the conditions given at the beginning of the second section. Therefore, $(|G| /|L(G)|) \geq 6$.

Now, without loss of generality, we may assume that $\left|C_{G}\left(\alpha_{1}\right)\right| \geq\left|C_{G}\left(\alpha_{2}\right)\right| \geq\left|C_{G}\left(\alpha_{3}\right)\right| \geq\left|C_{G}\left(\alpha_{4}\right)\right| . \quad$ Suppose $\left|C_{G}\left(\alpha_{1}\right)\right| \leq(|G| / 4)$; then, we have

$$
\begin{aligned}
|G| & =\left|C_{G}\left(\alpha_{1}\right)\right|+\left|C_{G}\left(\alpha_{2}\right)\right|+\left|C_{G}\left(\alpha_{3}\right)\right|+\left|C_{G}\left(\alpha_{4}\right)\right|-3|L(G)| \\
& \leq \frac{|G|}{4}+\frac{|G|}{4}+\frac{|G|}{4}+\frac{|G|}{4}-3|L(G)|=|G|-3|L(G)|,
\end{aligned}
$$

which is a contradiction. Hence, $\left|C_{G}\left(\alpha_{1}\right)\right|=(|G| / 2)$ or $(|G| / 3)$. If $\left|C_{G}\left(\alpha_{1}\right)\right|=(|G| / 2)$, we obtain

$$
\begin{aligned}
|G| & =\left|C_{G}\left(\alpha_{1}\right)\right|+\left|C_{G}\left(\alpha_{2}\right)\right|+\left|C_{G}\left(\alpha_{3}\right)\right|+\left|C_{G}\left(\alpha_{4}\right)\right|-3|L(G)| \\
& =\frac{|G|}{2}+\left|C_{G}\left(\alpha_{2}\right)\right|+\left|C_{G}\left(\alpha_{3}\right)\right|+\left|C_{G}\left(\alpha_{4}\right)\right|-3|L(G)| .
\end{aligned}
$$

One can easily calculate that

$$
\frac{|G|}{2}<\left|C_{G}\left(\alpha_{2}\right)\right|+\left|C_{G}\left(\alpha_{3}\right)\right|+\left|C_{G}\left(\alpha_{4}\right)\right| \leq 3\left|C_{G}\left(\alpha_{2}\right)\right|,
$$

so $(|G| / 6)<\left|C_{G}\left(\alpha_{2}\right)\right|$.

Now, applying (3) to $C_{G}\left(\alpha_{1}\right)$ and $C_{G}\left(\alpha_{2}\right)$, we have $\left(\left|C_{G}\left(\alpha_{1}\right)\right|\left|C_{G}\left(\alpha_{2}\right)\right| /|G|\right) \leq(|G| / 6), \quad$ and hence, $\left|C_{G}\left(\alpha_{2}\right)\right| \leq(2|G| / 6)$. That is, $(|G| / 6)<\left|C_{G}\left(\alpha_{2}\right)\right| \leq(|G| / 3)$, so $\left|C_{G}\left(\alpha_{2}\right)\right|=(|G| / 5),(|G| / 4)$ or $(|G| / 3)$. The property $\left(\left|C_{G}\left(\alpha_{1}\right)\right|\left|C_{G}\left(\alpha_{2}\right)\right| /|G|\right) \leq|L(G)| \leq(|G| / 6)$ implies that $(|G| / 10) \leq|L(G)| \leq(|G| / 6)$. Therefore, the value of $|L(G)|$ must be one of $(|G| / 6),(|G| / 7),(|G| / 8),(|G| / 9)$, or $(|G| / 10)$.

Now, if $|L(G)|=(|G| / 7)$, then $|L(G)|$ divides $\left|C_{G}\left(\alpha_{1}\right)\right|$, and hence, $2 \mid 7$ is impossible. Similarly, $|L(G)| \neq(|G| / 9)$. Hence, we have the following cases:

$$
|L(G)|=\frac{|G|}{6} \Rightarrow \frac{|G|}{|L(G)|}=6 \Rightarrow \frac{G}{L(G)} \cong C_{6} \text { or } S_{3} .
$$

If $(G / L(G)) \cong S_{3}$, then Theorem 3.5 of [9] and example 1 (iii) imply that $G \cong S_{3}$ and $\left|C_{S_{3}}(A)\right|=5$. Again Theorem 2.2 of [9] implies that if $(G / L(G)) \cong C_{6}$, then $G \cong C_{12}$, which does not conform to the conditions given at the beginning of Section 2.

Let $|L(G)|=(|G| / 8)$; then, as $|L(G)|$ divides $\left|C_{G}\left(\alpha_{2}\right)\right|$ if $\left|C_{G}\left(\alpha_{2}\right)\right|=(|G| / 3)$, then $3 \mid 8$, and if $\left|C_{G}\left(\alpha_{2}\right)\right|=(|G| / 5)$, then $5 \mid 8$, which both give contradictions. Therefore, $\left|C_{G}\left(\alpha_{2}\right)\right|=(|G| / 4)$. On the contrary, the property $|G|=\left|C_{G}\left(\alpha_{1}\right)\right|+\left|C_{G}\left(\alpha_{2}\right)\right|+\left|C_{G}\left(\alpha_{3}\right)\right|+\left|C_{G}\left(\alpha_{4}\right)\right|-3|L(G)|$ implies that $(|G| / 4)=\left|C_{G}\left(\alpha_{3}\right)\right|+\left|C_{G}\left(\alpha_{4}\right)\right|-3(|G| / 8)$, and hence, $\quad(5|G| / 8)=\left|C_{G}\left(\alpha_{3}\right)\right|+\left|C_{G}\left(\alpha_{4}\right)\right|$. As $\left|C_{G}\left(\alpha_{3}\right)\right|,\left|C_{G}\left(\alpha_{4}\right)\right| \leq(|G| / 4), \quad$ we obtain $(5|G| / 8)=\left|C_{G}\left(\alpha_{3}\right)\right|+\left|C_{G}\left(\alpha_{4}\right)\right| \leq(|G| / 2)$, which is again a contradiction. So, $|L(G)|$ cannot be equal to $(|G| / 8)$.

Finally, assume that $|L(G)|=(|G| / 10)$ and $|L(G)|$ divides $\left|C_{G}\left(\alpha_{2}\right)\right|$. If $\left|C_{G}\left(\alpha_{2}\right)\right|=(|G| / 3)$, then $3 \mid 10$, and if $\left|C_{G}\left(\alpha_{2}\right)\right|=(|G| / 4)$, then $4 \mid 10$, which are both impossible. Therefore, $\left|C_{G}\left(\alpha_{2}\right)\right|=(|G| / 5)$. Now, again $|G|=\left|C_{G}\left(\alpha_{1}\right)\right|+$ $\left|C_{G}\left(\alpha_{2}\right)\right|+\left|C_{G}\left(\alpha_{3}\right)\right|+\left|C_{G}\left(\alpha_{4}\right)\right|-3|L(G)|$ implies that 
$\left|C_{G}\left(\alpha_{3}\right)\right|+\left|C_{G}\left(\alpha_{4}\right)\right|=(6|G| / 10)$.

$\left|C_{G}\left(\alpha_{2}\right)\right| \geq\left|C_{G}\left(\alpha_{3}\right)\right| \geq\left|C_{G}\left(\alpha_{4}\right)\right|$ implies

Also, $(6|G| / 10)=\left|C_{G}\left(\alpha_{3}\right)\right|+\left|C_{G}\left(\alpha_{4}\right)\right|=(2|G| / 5)$, which is a contradiction. Hence, $|L(G)| \neq(|G| / 10)$.

Now, assume that $\left|C_{G}\left(\alpha_{1}\right)\right|=(|G| / 3)$. In this case, using

$$
|G|=\left|C_{G}\left(\alpha_{1}\right)\right|+\left|C_{G}\left(\alpha_{2}\right)\right|+\left|C_{G}\left(\alpha_{3}\right)\right|+\left|C_{G}\left(\alpha_{4}\right)\right|-3|L(G)|,
$$

we have

$(2|G| / 3)<\left|C_{G}\left(\alpha_{2}\right)\right|+\left|C_{G}\left(\alpha_{3}\right)\right|+\left|C_{G}\left(\alpha_{4}\right)\right| \leq 3\left|C_{G}\left(\alpha_{2}\right)\right|$.

Thus, $\quad\left|C_{G}\left(\alpha_{2}\right)\right|>(2|G| / 9)$. On the contrary, $\left|C_{G}\left(\alpha_{1}\right)\right| \geq\left|C_{G}\left(\alpha_{2}\right)\right|$, so $(2|G| / 9)<\left|C_{G}\left(\alpha_{2}\right)\right| \leq(|G| / 3)$. Therefore, $\left|C_{G}\left(\alpha_{2}\right)\right|=(|G| / 3)$ or $(|G| / 4)$. Again applying (*) on $C_{G}\left(\alpha_{1}\right)$ and $C_{G}\left(\alpha_{2}\right)$, we obtain

$$
\frac{\left|C_{G}\left(\alpha_{1}\right)\right|\left|C_{G}\left(\alpha_{2}\right)\right|}{|G|} \leq|L(G)| \leq \frac{|G|}{6} .
$$

Thus, $\quad(|G| / 12) \leq|L(G)| \leq(|G| / 6), \quad$ and hence, $|L(G)|=(|G| / 6),(|G| / 7)$,

(|G|/8), (|G|/9), (|G|/10), (|G|/11), or $(|G| / 12)$.

Assume that $|L(G)|=(|G| / 7)$, and as $|L(G)|$ divides $\left|C_{G}\left(\alpha_{1}\right)\right|$, we must have $3 \mid 7$, which is impossible. Similarly, $|L(G)| \neq(|G| / 8),(|G| / 10)$, and $(|G| / 11)$. Also, assume that $|L(G)|=(|G| / 6), \quad\left|C_{G}\left(\alpha_{1}\right)\right|=(|G| / 3), \quad$ and $\left|C_{G}\left(\alpha_{2}\right)\right|=(|G| / 4)$ or $(|G| / 3)$; then, again

$$
|G|=\left|C_{G}\left(\alpha_{1}\right)\right|+\left|C_{G}\left(\alpha_{2}\right)\right|+\left|C_{G}\left(\alpha_{3}\right)\right|+\left|C_{G}\left(\alpha_{4}\right)\right|-3|L(G)|
$$

implies that $(11|G| / 12)=\left|C_{G}\left(\alpha_{3}\right)\right|+\left|C_{G}\left(\alpha_{4}\right)\right| \leq(|G| / 2)$ or $(5|G| / 6)=\left|C_{G}\left(\alpha_{3}\right)\right|+\left|C_{G}\left(\alpha_{4}\right)\right| \leq(2|G| / 3), \quad$ respectively, which are both impossible. Hence, $|L(G)| \neq(|G| / 6)$, so we have one of the following cases:

$$
|L(G)|=\frac{|G|}{12} \Rightarrow \frac{|G|}{|L(G)|}=12 \Rightarrow \frac{G}{L(G)} \cong C_{12}, A_{4}, D_{12}, C_{3} \rtimes C_{4}, C_{2} \times C_{6},
$$

or

$$
|L(G)|=\frac{|G|}{9} \Rightarrow \frac{|G|}{|L(G)|}=9 \Rightarrow \frac{G}{L(G)} \cong C_{9}, C_{3} \times C_{3} .
$$

\section{Groups with Abelian A-Centralizers}

The concept of commutative transitive groups was first introduced and studied by Weisner [11] in 1925.

In this section, we introduce the new concept of autocommutative transitive groups, which is a generalized version of commutative transitive groups. Also, we study a group $G$, in which every $A$-centralizer of a nontrivial element of $G$ is abelian. We show that such groups are equivalent to autocommutative transitive groups.

Definition 1. A group $G$ is autocommutative transitive (henceforth $A-\mathrm{CT}$ ) if $[x, \alpha]=1$ and $[\alpha, y]=1$ imply that $[x, y]=1$, for any nontrivial elements $x, y$ in $G$ and every $\alpha \in \mathrm{A}$.
If $\alpha$ runs over the inner automorphisms of $G$, then one has the usual commutative transitive groups.

Lemma 3. For any group $G$, the following statements are equivalent:

(i) $G$ is A-CT group

(ii) The A-centralizers of nontrivial automorphisms of $G$ are abelian

Proof

(i) $\Rightarrow$ (ii) Let $G$ be A-CT group. For any nonidentity automorphism element $\alpha \in \mathrm{A}$, if $x, y \in C_{G}(\alpha)$, we have $[x, \alpha]=1$ and $[\alpha, y]=1$. The definition of A-CT implies that $[x, y]=1$. Hence, $C_{G}(\alpha)$ is abelian.

(ii) $\Rightarrow$ (i) Assume $x, y$ are nontrivial elements of $G$, with $[x, \alpha]=1$ and $[\alpha, y]=1$, for every $\alpha \in$ A. Obviously, $x, y \in C_{G}(\alpha)$, by the assumption $C_{G}(\alpha)$ is abelian, and hence, $[x, y]=1$. Thus, $G$ is autocommutative transitive.

Using the above lemma, we have the following.

Corollary 1. Let $G$ be a finite A-CTgroup and $\left\{x_{1}, \ldots, x_{r}\right\}$ be a set of pairwise noncommuting elements of $G$ with maximal size. Then, $\left|C_{G}(A)\right| \geq r+1$.

\section{Data Availability}

The datasets used and analysed during the study are included within the article.

\section{Conflicts of Interest}

The authors declare that they have no conflicts of interest.

\section{References}

[1] S. Davoudirad, M. R. R. Moghaddam, and M. A. Rostamyari, "Autonilpotent groups and their properties," Asian-European Journal of Mathematics, vol. 9, no. no. 2, Article ID 1650056, 2016.

[2] P. Hegarty, “The absolute center of a group," Journal of Algebra, vol. 169, no. 3, pp. 929-935, 1994.

[3] P. V. Hegarty, "Autocommutator subgroups of finite groups," Journal of Algebra, vol. 190, no. 2, pp. 556-562, 1997.

[4] M. R. R. Moghaddam, F. Parvaneh, and M. Naghshineh, "The lower autocentral series of abelian groups," Bulletin of the Korean Mathematical Society, vol. 48, no. 1, pp. 79-83, 2011.

[5] F. Parvaneh and M. R. R. Moghaddam, "Some properties of autosoluble groups," Journal of Mathematical Extension, vol. 5, no. 1, pp. 13-19, 2010.

[6] M. M. Nasrabadi and A. Gholamian, "On finite n-acentralizer groups," Communications in Algebra, vol. 43, no. 2, pp. 378-383, 2015.

[7] S. M. Belcastro and G. J. Sherman, "Counting centralizers in finite groups," Mathematics Magazine, vol. 67, no. 5, pp. 366-374, 1994. 
[8] A. R. Ashrafi, "On finite groups with a given number of centralizers," Algebra Colloquium, vol. 7, no. 2, pp. 139-146, 2000.

[9] M. Chaboksavar, M. Farrokhi Derakhshandeh Ghouchan, and F. Saeedi, "Finite groups with a given absolute central factor group," Archiv der Mathematik, vol. 102, no. 5, pp. 401-409, 2014.

[10] M. Bruckheimer, A. C. Bryan, and A. Muir, "Groups which are the union of three subgroups," The American Mathematical Monthly, vol. 77, no. no. 1, pp. 52-57, 1970.

[11] L. Weisner, "Groups in which the normaliser of every element except identity is abelian," Bulletin of the American Mathematical Society, vol. 31, no. 8, pp. 413-416, 1925. 